\title{
Caminhos possíveis para a avaliação das práticas da Vigilância em Saúde
}

\author{
Possible ways for Public Health Surveillance practices evaluation
}

Maria Filomena de Gouveia Vilela ${ }^{1}$

Dario Nunes dos Santos ${ }^{2}$

Brigina Kemp ${ }^{1}$

\footnotetext{
${ }^{1}$ Faculdade de Enfermagem, Universidade Estadual de Campinas. Cidade Universitária Zeferino Vaz, Barão Geraldo. 13083970 Campinas SP Brasil. mena@unicamp.br ${ }^{2}$ Departamento de Vigilância em Saúde, Prefeitura Municipal de Guarulhos. Guarulhos SP Brasil.
}

\begin{abstract}
This is an evaluative and qualitative study that proposes to investigate self-assessment evaluation as a device to analyze Health Surveillance practices through a questionnaire built by researchers, adapted from the Self-Assessment of Improved Access and Primary Care Quality (AMAQ) and available on the FORMSUS platform. Forty-one Health Surveillance workers and managers of a large municipality from São Paulo State evaluated the realms of "management", "teamwork" and their respective sub-realms. Two categories were created to analyze the results: "Management" and "Team" in dialogue with references from Management, Evaluation and Health Surveillance. Most "management" and "teamwork" sub-realms were deemed satisfactory. Self-assessment evaluation through an applied evaluation tool was shown to be a powerful resource for the analysis of Health Surveillance practices in combination with other devices adopted by the Unified Health System (SUS). Unlike usual evaluation processes guided by quantitative markers, this self-assessable evaluative process included subjects and enabled the possibility of incorporating a new look at itself to the way Health Surveillance is carried out and support future management contracts between workers and managers.

Key words Public Health Surveillance, Health services evaluation, Health services administration
\end{abstract}

Resumo Trata-se de pesquisa avaliativa, qualitativa que se propôs investigar a autoavaliação como dispositivo de análise das práticas da Vigilância em Saúde, valendo-se de questionário construído pelos pesquisadores, adaptado da Autovaliação da Melhoria do Acesso e da Qualidade da Atenção Básica (AMAQ) e disponibilizado na plataforma FORMSUS. Quarenta e um trabalhadores e gestores da Vigilância em Saúde de um município de grande porte do estado de São Paulo avaliaram as dimensões da "gestão" e "trabalho da equipe" e suas respectivas subdimensões. Criaram-se duas categorias de análise dos resultados: "Gestão" $e$ "Equipe", em diálogo com referenciais da Gestão, Avaliação e Vigilância em Saúde. A maior parte das subdimensões da "gestão" e "trabalho da equipe" foram avaliadas como satisfatórias. A autoavaliação, mediante o instrumento aplicado, mostrou-se potente para a análise das práticas da Vigilância em Saúde, em conjunto com outros dispositivos adotados no SUS. Diferentemente dos processos habituais de avaliação, pautados em indicadores quantitativos, este processo autoavaliativo incluiu os sujeitos e trouxe a possibilidade de incorporar aos modos de fazer da Vigilância em Saúde o olhar para si e sustentar futuros contratos de gestão entre trabalhadores e gestores.

Palavras-chave Vigilância em saúde pública, Avaliação de serviços de saúde, Administração de serviços de saúde 


\section{Introdução}

Este estudo investigou a autoavaliação como dispositivo de análise das práticas da Vigilância em Saúde (VS). Aplicou-se questionário autoavaliativo a gestores e trabalhadores do Departamento de Vigilância em Saúde (DVS) da Secretaria Municipal de Saúde de Guarulhos (SP), adaptado da Autoavaliação da Melhoria do Acesso e da Qualidade (AMAQ), parte integrante do Programa de Melhoria do Acesso e da Qualidade da Atenção Básica (PMAQ) do Ministério da Saúde (MS) ${ }^{1,2}$.

A motivação para o estudo partiu da existência de processos avaliativos e de contratos de trabalho entre gestores e trabalhadores naquele município, a exemplo do PMAQ e do Programa de Qualificação das Ações de Vigilância em Saúde (PQA-VS) $)^{3,4}$.

Em geral, ações de VS têm sido avaliadas a partir de resultados alcançados, tendo como referência metas pactuadas entre os entes federados. Em geral, utilizam-se indicadores epidemiológicos para demonstrar a capacidade de controle de doenças ou agravos, ou ainda, número de inspeções, no caso da Vigilância Sanitária ${ }^{3,5}$.

Buscou-se, nesta investigação, outros referenciais, na tentativa de compreender a avaliação como processo formativo, no qual se leve em conta a opinião dos sujeitos das práticas e que possa ser incorporada também como gestão do trabalho em Vigilância ${ }^{6,7}$.

O PMAQ tem suscitado debates e investigações, com apontamentos de acertos e equívocos desse tipo de avaliação, no qual o repasse de recursos fica condicionado à adequação de parâmetros de qualidade ${ }^{8,9}$.

De todo modo, o componente da autoavaliação é entendido como importante e ponto de partida de desenvolvimento do PMAQ, pois permite o reconhecimento das dimensões positivas e também problemáticas da organização do processo de trabalho e da gestão das equipes. Identificam-se, a partir de uma dinâmica pedagógica, nós críticos que dificultam o desenvolvimento das ações de saúde no território, bem como conquistas alcançadas pelas intervenções implementadas. A AMAQ foi baseada em várias outras ferramentas de avaliação, utilizadas no Brasil e em outros países ${ }^{1,2}$

Alguns autores têm se ocupado do estudo de processos avaliativos que, se orientados pela inclusão e participação dos diversos atores envolvidos, tornam-se dispositivos de mudanças nas instituições ${ }^{6,10}$. Segundo Furtado ${ }^{6}$, é necessário incluir as diferentes concepções a partir do ponto de vista dos grupos envolvidos com um programa ou serviço, uma vez que o senso comum e a avaliação tradicional não dão conta de abranger todos os aspectos da complexidade das iniciativas de enfrentamento de problemas sociais e de saúde.

A VS é um vasto campo de saberes e práticas do Sistema Único de Saúde (SUS) e o mais recente marco legal, a Portaria $1.378^{11}$ a define como:

um processo contínuo e sistemático de coleta, consolidação, análise e disseminação de dados sobre eventos relacionados à saúde, visando o planejamento e a implementação de medidas de saúde pública para a proteção da saúde da população, a prevenção e controle de riscos, agravos e doenças, bem como para a promoção da saúde.

A referida portaria, afirma que "as ações de Vigilância em Saúde abrangem toda a população brasileira” e define seus núcleos de saberes e práticas voltados para a vigilância de doenças transmissíveis, doenças crônicas não transmissíveis, entre outros agravos e condições de saúde. Não discrimina especificamente os núcleos das vigilâncias epidemiológica e ambiental; refere-se ainda à "Vigilância Sanitária dos riscos decorrentes da produção e do uso de produtos, serviços e tecnologias de interesse à saúde" 11 .

Esta definição se aproxima da formulação de Vigilância em Saúde Pública, termo utilizado em outros países para denominar a "nova Vigilância Epidemiológica”, a fim de não haver confusão com a Epidemiologia, disciplina que dá base para a prática das várias "vigilâncias"12,13.

A Lei $8.080^{14}$ definiu os conceitos de Vigilância Epidemiológica e Vigilância Sanitária, práticas já consolidadas, que se confundem com a própria história da Saúde Pública no Brasil ${ }^{15}$. Naquele momento de implantação do SUS, alguns autores da Saúde Coletiva brasileira formularam um novo modelo de atenção, uma nova prática sanitária, a "Vigilância da Saúde", ou "Vigilância à Saúde", que de alguma maneira incorporou as práticas da Vigilância Sanitária e da Vigilância Epidemiológica intervindo sobre as causas, os riscos e os danos, como proposto no modelo ${ }^{16-18}$.

Essa polissemia de termos similares no Brasil gera certa confusão entre gestores e trabalhadores, que, desde o advento do SUS, vêm tentando institucionalizar a VS nos estados e municípios, como um campo, com seus núcleos distintos de saberes e práticas ${ }^{19}$, que inclui a Vigilância Epidemiológica (VE), a Vigilância Sanitária (Visa), a Vigilância da Saúde do Trabalhador (VST) e a Vigilância em Saúde Ambiental (VA) ${ }^{15,20,21}$.

Esse é um terreno de disputas conceituais e de modelos, sobretudo quando se trata da Vigilân- 
cia Sanitária. Esta tem sido estudada por alguns autores, que a consideram uma prática da Saúde Pública, com seu objeto bem definido, articulada com as demais "vigilâncias"22,23. Outros a tem considerado como um "território vizinho" à nova Vigilância em Saúde Pública, como concebida no Brasil desde a criação da Secretaria de Vigilância em Saúde do MS, em $2003^{24}$.

De todo modo, o MS, ao editar a Portaria $1.378^{11}$, resolveu, em seu capítulo IV, artigo 42 , indicar a criação de um Grupo de Trabalho (GT) para elaborar a Política Nacional de Vigilância em Saúde (PNVS) e o fez pela Portaria SVS/MS $n^{\circ} 14 / 2013^{25}$. O GT-PNVS foi composto por integrantes da Secretaria de Vigilância em Saúde (SVS) do MS, Agência Nacional de Vigilância Sanitária (Anvisa), Conselho Nacional de Secretários Estaduais de Saúde (CONASS) e Conselho Nacional de Secretários Municipais de Saúde (CONASEMS).

O referido GT elaborou um "Documento Base", preliminar ${ }^{26}$, objeto de debate no Curso de Vigilância em Saúde, realizado no XXIX Congresso de Secretários Municipais de Saúde do Estado de São Paulo, em 2015, organizado pelo Conselho de Secretários Municipais de Saúde (COSEMS) ${ }^{27}$. Uma das representantes do MS, palestrante no referido curso, apresentou a proposição do GT em ampliar o conceito de VS, incorporando a questão da "regulação sanitária, a integralidade das ações e a integração das vigilâncias" 28 .

Apesar da elaboração do Documento Base, no decorrer do ano de 2015, não progrediu a discussão da Política de Vigilância em Saúde no país. Em maio de 2016, o MS publicou a Portaria GM/MS no. 1.017 de 11 de maio de 2016 com o objetivo de propor diretrizes para a formulação da PNVS e o fortalecimento dos programas e ações de $\mathrm{VS}^{29}$. Ainda em 2016, o Ministério publicou a Resolução no 535, de 19 de agosto de 2016, na qual define o regimento da Conferência Nacional de Vigilância em Saúde, a ser realizada em novembro de $2017^{30}$. Este é um momento rico para o debate, no qual, gestores, trabalhadores e usuários poderão discutir os conceitos, a Política, os modelos e as práticas da VS.

Apesar de preliminar, o conteúdo do Documento Base $\mathrm{B}^{26}$, especialmente no que se refere aos princípios, diretrizes e eixos organizativos da futura Política, assim como outros documentos e marcos legais da VS, serviram de inspiração para a construção das subdimensões presentes no questionário elaborado para este estudo.

\section{Métodos}

Trata-se de pesquisa avaliativa, de abordagem qualitativa que se utilizou de questionário elaborado para a autoavaliação de gestores e trabalhadores sobre conteúdos associados às práticas da VS. A pesquisa ocorreu em Guarulhos, município da Região Metropolitana de São Paulo e segunda maior cidade do estado ${ }^{4}$.

Os sujeitos da pesquisa foram trabalhadores e gestores do DVS. De 202 profissionais convidados por e-mail, 41 (20\%) aceitaram, sendo 13 do Centro de Controle de Zoonoses, 1 do Serviço de Verificação de óbito, 9 da Vigilância Epidemiológica, 2 da Diretoria; 3 do Laboratório de Saúde Pública, 12 da Vigilância Sanitária e 1 gestor municipal.

O instrumento foi adaptado do programa de Avaliação para Melhoria da Qualidade da Estratégia da Saúde da Família (AMQ e AMAQ) e de outras ferramentas de avaliação de serviços de saúde, utilizadas e validadas nacional e internacionalmente $^{1,2}$. A pesquisa avaliativa deve incluir diversas visões e valores, viabilizar e ampliar a utilização dos recursos da avaliação, considerar o inevitável caráter político da pesquisa em geral e da avaliativa em particular e capacitar os envolvidos com o programa ou serviço avaliado ${ }^{6}$. Além disso, tem como objetivo produzir conhecimento que poderá orientar decisões sobre viabilidade, disponibilidade de tempo e de recursos a serem aplicados em determinadas áreas ou setores ${ }^{10}$.

O questionário autoavaliativo foi concebido a partir de 4 dimensões: gestão municipal, gestão da VS, gestão do serviço de vigilância e equipe da Vigilância em Saúde (perfil e processo de trabalho da equipe), que se desdobram em 14 subdimensões e, estas, em questões (padrões) que abrangem o que é esperado em termos de qualidade para a VS. Foram 110 questões, 50 destas dirigidas a gestores; 28 direcionadas aos dois (gestores e trabalhadores); 32 aos trabalhadores, seguindo as competências da gestão, coordenação e equipes de VS.

No instrumento constavam questões fechadas e abertas, validado em fase pré-teste por gestores do DVS e um gestor da Secretaria de Saúde do município. Foi respondido online, na plataforma FORMSUS (serviço de uso público, disponibilizado pelo DATASUS para a criação de formulários na WEB), onde o respondente acionava o conteúdo do Termo de Consentimento Livre e Esclarecido e assinalava a concordância em participar da pesquisa. Os dados foram transpostos 
para um banco de dados em Excel para consolidação dos resultados.

Para as questões fechadas, o trabalhador ou o gestor atribuía uma nota aos padrões esperados para as subdimensões. Essas notas eram consolidadas com base em escala de valores que representam os padrões acerca de perguntas ou declarações referentes à qualidade esperada quanto à estrutura, aos processos e resultados da VS. As notas variavam de zero a dez para cada padrão e a escala de valores produzida variou entre classificação de "muito insatisfatório", "insatisfatório", "regular", "satisfatório" e "muito satisfatório", conforme a média das notas atribuídas pelo conjunto dos respondentes.

Além disso, o respondente tinha disponível um espaço de registro de suas opiniões sobre o item avaliado, o que definiu o caráter qualitativo do estudo. Essas respostas abertas foram analisadas pela técnica de análise de conteúdo temáti$\mathrm{ca}$, que, segundo Minayo ${ }^{31}$ e Bardin ${ }^{32}$ consiste em descobrir os núcleos de sentido que compõem uma comunicação, cuja presença ou frequência signifique alguma coisa para o objeto estudado. As dimensões foram tomadas como unidades de análise e as subdimensões como unidades temáticas. Para a fase de análise dos resultados foram criadas duas categorias analíticas: "Gestão" - subdividida em gestão municipal, da VS e dos serviços de vigilância e "Equipe".

Esta pesquisa foi aprovada pelo Comitê de Ética em Pesquisa da Universidade Estadual de Campinas.

\section{Resultados e Discussão}

Os resultados demonstram que há apreciação satisfatória das práticas da VS (Quadro 1).

A seguir, será apresentada a análise e a discussão dos resultados a partir das categorias da "Gestão" e "Equipe", em diálogo com alguns referenciais teóricos da Gestão e Avaliação em Saúde e os marcos legais do SUS e da VS.

\section{Gestão}

Gestão Municipal - subdimensões de A a D. Os resultados dizem respeito ao conjunto das ações da gestão municipal para garantir que a VS cumpra seu papel de proteção da saúde dos cidadãos, atribuído pelos marcos legais do SUS ${ }^{11,33}$.

As questões relativas ao papel da gestão municipal de implantar e implementar a VS e sua

Quadro 1. Subdimensão por padrões de qualidade, pontuação, resultado e classificação.

\begin{tabular}{|l|c|c|c|c|l|}
\hline \multicolumn{1}{|c|}{ Subdimensões } & $\begin{array}{c}\text { Número } \\
\text { padrões }\end{array}$ & $\begin{array}{c}\text { Pontuação } \\
\text { (mínima) }\end{array}$ & $\begin{array}{c}\text { Pontuação } \\
\text { (máxima) }\end{array}$ & Resultado & \multicolumn{1}{|c|}{ Classificação } \\
\hline $\begin{array}{l}\text { A. Implantação e implementação da } \\
\text { Vigilância em Saúde }\end{array}$ & 18 & 0 & 180 & 133 & Satisfatório \\
\hline $\begin{array}{l}\text { B. Organização e integração da rede } \\
\text { de atenção à saúde }\end{array}$ & 5 & 0 & 50 & 35 & Satisfatório \\
\hline C. Gestão do trabalho & 4 & 0 & 40 & 18 & Regular \\
\hline $\begin{array}{l}\text { D. Participação, controle social e } \\
\text { satisfação do usuário }\end{array}$ & 4 & 0 & 40 & 34 & Muito satisfatório \\
\hline E. Apoio institucional & 9 & 0 & 90 & 63 & Satisfatório \\
\hline F. Educação Permannete & 6 & 0 & 60 & 43 & Satisfatório \\
\hline $\begin{array}{l}\text { G. Gestão do monitoramento e } \\
\text { avaliação }\end{array}$ & 7 & 0 & 70 & 31 & Satisfatório \\
\hline H. Infraestrutura e equipamentos & 5 & 0 & 50 & 29 & Regular \\
\hline $\begin{array}{l}\text { I. Insumos, imunobiológicos e } \\
\text { medicamentos }\end{array}$ & 16 & 0 & 160 & 103 & Satisfatório \\
\hline J. Sistemas de informação & 3 & 0 & 30 & 18 & Satisfatório \\
\hline K. Perfil da equipe & 14 & 0 & 140 & 89 & Regular \\
\hline $\begin{array}{l}\text { L. Organização do Processo de } \\
\text { trabalho }\end{array}$ & 4 & 0 & 40 & 30 & Satisfatório \\
\hline M. Atenção integral à saúde & 0 & 110 & 75 & Satisfatório \\
\hline $\begin{array}{l}\text { N. Participação, controle social e } \\
\text { satisfação do usuário }\end{array}$ & 11 & 0 & 0 & & \\
\hline
\end{tabular}


competência referente à coordenação do componente municipal dos Sistemas Nacionais de VS e de Visa trazem à tona a necessidade de discussão de uma Política de VS para o país. Estabelecer um conceito, valores, princípios, diretrizes e prioridades de uma política pode facilitar o trabalho da gestão, ao indicar um norte e deixar mais claras suas atribuições, em geral relevantes e complexas.

A formulação de uma política deve responder aos interesses dos diversos grupos e classes sociais para o atendimento da necessidade social identificada em determinado momento histórico e político ${ }^{34}$. No caso da Política de VS, há vários interesses em disputa e poderá ficar mais claro aos gestores de qual VS se está falando: aquela que se aproxima de um conceito de Vigilância em Saúde Pública ou VE, como definido na Portaria $1.378^{11}$, ou um conceito mais amplo que engloba no mesmo campo todas as vigilâncias?

Espera-se dos gestores a realização de análises de situação de saúde centradas em danos, riscos, condicionantes e determinantes socioambientais, possibilitando a garantia de proteção da saúde da população, a prevenção e o controle de riscos, agravos e doenças, além da promoção da saúde. Tarefas que não são exclusivas dos profissionais "especialistas" das vigilâncias, mas também de outros atores dos serviços de saúde, particularmente da Atenção Básica ${ }^{35}$.

A autoavaliação dos gestores foi positiva em relação à capacidade da gestão de preparar ações de vigilância nas emergências de saúde pública; coordenar, monitorar e fazer avaliação da estratégia sentinela em âmbito hospitalar e desenvolver estratégias para vigilância dos riscos e agravos de forma articulada na rede de atenção à saúde, pois as ações de vigilância, mesmo que coordenadas regionalmente ou centralmente nos municípios, executa e apoia ações nos territórios. Foi também positiva a garantia do envolvimento da VS nas redes temáticas, além de assegurar estratégias de integração das práticas e processo de trabalho das vigilâncias. Desafiadoras estas novas funções da gestão em implantar e garantir articulação nas novas redes temáticas ${ }^{36}$.

Recente estudo sobre a implantação da Rede Cegonha revelou dificuldade de integração das práticas da Visa e área de saúde da mulher na região de Campinas (SP). A fragmentação é uma tônica apontada pelos gestores da VS, tanto interna quanto externamente ${ }^{37}$. Investimentos em dispositivos de gestão, que valorizem os espaços coletivos, a cogestão e o apoio matricial são estratégias para diminuir os "estranhamentos" e desconhecimentos existentes entre as áreas que compõem o SUS, a VS entre elas ${ }^{38,39}$. A segurança do paciente pode ser uma das ofertas da Vigilância Sanitária (Visa) aos serviços de saúde da rede municipal, na linha da educação permanente.

Os tópicos relacionados à gestão do trabalho dizem respeito à política de valorização dos trabalhadores, como a existência de plano de cargos, carreiras e salários, e premiação das equipes, seja por desempenho, cumprimento de metas pactuadas ou proteção do trabalhador. Problemas estruturais, alguns agravados por decisões dos governos que estão no comando, somados ao agravamento do financiamento do SUS anunciado recentemente ${ }^{40}$, geram um cenário incômodo para qualquer gestor municipal ou da VS, como expressado por alguns:

Falta garantir a quantidade de funcionários suficientes para então manter uma qualidade de prestação de serviço. Há uma sobrecarga de trabalho

Mesmo um financiamento mais robusto para a área de VS não conseguirá fazer frente a essas lacunas estruturais e institucionais apresentadas nas várias políticas sociais. Ainda assim, deixar públicos estes problemas e colocá-los em análise em gestões democráticas e participativas pode ser uma forma de buscar soluções locais.

O tema da participação, controle social e satisfação do usuário foi avaliado como "muito satisfatório". Consideraram que a gestão promove e assegura aos cidadãos o acesso às informações e à participação na formulação, implementação $\mathrm{e}$ avaliação das políticas de saúde, considerando o direito à saúde e à cidadania.

Do ponto de vista da VS, amiúde a comunicação e o diálogo com a sociedade são conflituosos, particularmente na Visa, pois se confrontam interesses do mercado e do Estado, este com função de proteção da saúde ${ }^{23}$. Estudo recente abordou a comunicação social durante conflito com moradores devido a intervenções da VS no controle de leishmaniose visceral em cães, ao indicar a eutanásia como uma das medidas direcionadas a animais doentes ${ }^{41}$. Conflitos não devem ser barreiras nessa comunicação. A 1a Conferência Nacional de VS pode ser mais um passo nesta aproximação entre gestores, trabalhadores e usuários.

Gestão da Vigilância em Saúde - Subdimensões E a G. O exercício do Apoio Institucional foi relatado por gestores como tecnologia de gestão para auxiliar equipes a construir grupalidade, analisar o próprio trabalho e construir intervenções. Segundo Oliveira e Campos ${ }^{39}$, apoio Institucional é uma "metodologia que se caracteriza por reformular os mecanismos tradicionais da gestão, ao adotar uma postura interativa, ao mesmo 
tempo analítica e operacional, que complementaria e transformaria a forma de desempenhar as funções gerenciais como a de uma coordenação".

Interessante analisar se a prática do Apoio Institucional persiste, pois juntamente com os demais dispositivos, como a da Educação Permanente, parece fortalecer a gestão, segundo os atores da autoavaliação.

Os gestores consideraram a prática do monitoramento e avaliação satisfatória, apesar dos limites institucionais. Utilizam processos avaliativos como ferramenta de gestão; adotam estratégias que fortalecem a alimentação e o uso de sistemas de informações; organizam e adotam ações de monitoramento no âmbito da vigilância; promovem a discussão de resultados e estimulam o uso da informação pelas equipes de VS. Consideram ainda que cumprem os pactos estabelecidos com outros entes federativos, conforme os resultados alcançados, como boas coberturas vacinais e avanços em alguns programas como IST, Aids e Tuberculose ${ }^{4}$.

O uso da informação e sua incorporação no cotidiano dos gestores e das equipes, incluindo o monitoramento e a avaliação, são cruciais para a prática da VS. Pode-se dizer que é o cerne, o que subsidia os planejamentos, fundamenta e orienta as intervenções de saúde. Resta saber como se utiliza a informação: apenas nos planejamentos anuais e para os "macro-diagnósticos tardios"42, monitoramentos e avaliações esporádicas e burocráticas, ou realmente faz parte do dia a dia dos gestores e trabalhadores da VS para direcionar o trabalho, voltado a captar e atender as necessidades de saúde da população? Neste processo autoavaliativo foi possível perceber a diferença do olhar do gestor e do trabalhador, principalmente os da Visa, que relatam a quase ausência do uso da informação, monitoramento e avaliação para subsidiar as ações cotidianas.

Gestão do Serviço de Vigilância - subdimensões $\mathrm{H}$ a J foram respondidas por gestores e trabalhadores que reconhecem como deficitária a infraestrutura física e de equipamentos, o que dificulta o desempenho das atividades cotidianas:

Atendemos os clientes na frente de todos os profissionais ou no corredor ou na sala de reunião quando não está sendo utilizada.

Avaliou-se ainda aspectos relativos ao armazenamento, disponibilidade e suficiência de insumos, imunobiológicos e medicamentos para o desenvolvimento de ações de Vigilância e processo de dispensação e controle das vacinas, kits de laboratório e medicamentos pelos serviços de saúde.
Os atores apontaram dificuldades do gestor em ofertar condições de trabalho relacionadas às questões estruturais. Ainda assim, houve uma avaliação satisfatória do desempenho das ações da vigilância como um todo, muitas vezes realizadas pelo esforço individual e com recursos próprios dos profissionais.

Fatores discutidos neste tópico permitem refletir sobre como a gestão municipal da VS pode proporcionar condições de trabalho para o desenvolvimento de ações sob seu encargo, cada vez mais complexas, exigindo, portanto, infraestrutura adequada para enfrentar situações cotidianas e emergenciais ${ }^{43}$. O que demanda financiamento adequado, além de questões administrativas e gerenciais locais que permitam operacionalizar aquisições necessárias e de manutenção. Ao longo dos últimos anos, são identificadas várias iniciativas para fortalecer e qualificar a gestão e o financiamento da VS, como, por exemplo, o Vigisus etapas I e II ${ }^{44,45}$.

Brito $^{46}$, em estudo avaliativo do Projeto Vigisus aponta a pequena governabilidade da área da VS para intervir em aspectos administrativos e gerenciais do governo municipal. É necessario debater a capacidade organizativa de executar o financiamento, o que pressupõe apoio entre os entes federativos, considerando questões burocráticas para agilizar os investimentos no setor.

Sobre os sistemas de informação, foram abordados pontos relacionados à qualidade da informação produzida, conhecimento das equipes sobre os sistemas vigentes, alimentação e estratégias adotadas para seu fortalecimento, além do uso e divulgação das informações produzidas.

A despeito da autoavaliação ter sido considerada satisfatória, as observações feitas apontam questões para reflexão sobre o processo de trabalho, tanto dos gestores quanto das equipes. Para além das especificidades locais, este é um tema desafiador para a VS. São muitos os sistemas de informação em saúde existentes, alguns exclusivos da VS.

Os sistemas de informações sobre eventos vitais, nascidos vivos (SINASC) e mortalidade (SIM) são importantes para o desenvolvimento das atividades de vigilância e, conforme a organização das secretarias municipais, são alocados ora na VS, ora em outros setores ${ }^{47,48}$.

As falas revelaram fragmentação do trabalho na utilização de cada sistema de informação, afastamento e certo desconhecimento em relação aos bancos de informações de eventos vitais. Sobre o uso das informações pela gestão, perguntam:

que monitoramento, que avaliação? 
Em relação à utilização de dados e informações produzidos pela vigilância e seu uso em Unidades Básicas:

as informações produzidas pela vigilância, nem sempre são utilizadas nas Unidades Básicas de Saúde... nem em outros serviços de saúde...

Alguns trabalhadores apontam que os gestores cobram produção, a "pilha de processos em cima da mesa" e as vistorias realizadas, ao invés de informação para direcionar o atendimento às necessidades de saúde:

o que se preocupa no setor onde estou é com números de processos atendidos

A existência de vários sistemas de informação, falta de padronização de coleta e tratamento dos dados, problemas com conectividade e dificuldade em capacitações são de conhecimento do MS, traduzidos na Política de Informação e Informática, com vistas a superar estes problemas e avançar em ampla utilização de informações no SUS ${ }^{48}$.

Os sistemas de informação em saúde possibilitam coletar dados, armazenar e organizar de modo a viabilizar monitoramento e análise para identificar riscos e problemas de saúde a fim de permitir o conhecimento do estado de saúde de uma população ${ }^{49}$, o que denota a importância da produção e do uso da informação no campo da VS como subsídio para a tomada de decisões nos diversos níveis da federação.

\section{Equipe}

Compõe as subdimensões $\mathrm{K}, \mathrm{L}, \mathrm{M}, \mathrm{N}$, avaliadas pelos trabalhadores. No que se refere à Educação Permanente e qualificação das equipes da VS, os trabalhadores apontaram resultado "satisfatório", apesar de certa insuficiência em atender os requisitos de competências e desenvolvimentos de habilidades dos profissionais, pois não foi bem avaliada a formação complementar das equipes, participação em cursos de atualização, bem como o uso de dispositivos de educação e apoio matricial à distância para a qualificação do cuidado prestado aos usuários, apesar do reconhecimento do esforço da gestão:

Os cursos de atualização e qualificação não são oferecidos a todos os profissionais da Vigilância Sanitária [...] entendo que são poucas as vagas, o que dificulta a melhoria do trabalho e qualificação....

A Organização dos Processos de Trabalho foi avaliada como "regular". Os quesitos abordavam: trabalho pautado em prioridades, classificação de risco e vulnerabilidades, envolvimento da sociedade no planejamento de ações, acesso ao muní- cipe, articulação intersetorial, atuação sobre determinantes e condicionantes, monitoramento e avaliação e realização de reuniões periódicas.

Aspectos conceituais que alicerçam as práticas das vigilâncias subsidiam esta avaliação e podem ser objeto de educação permanente, também avaliada como regular pelos profissionais. Percebe-se fragilidade nestes conhecimentos por parte das equipes:

alguns profissionais não sabem nem o que são os determinantes sociais

Um desafio para gestores da VS é organizar um modo de trabalhar pautado em risco, no território, indicadores epidemiológicos, planejamento, monitoramento e avaliação, além de aspectos relacionados às estratégias de gestão. Segundo os trabalhadores, há falta de espaço para reflexão sobre o serviço, não obstante avanços no trabalho da equipe quando há participação dos mesmos no planejamento:

Há muito o que melhorar, mas o planejamento das ações com a maior participação dos funcionários definindo as prioridades, elevou a qualidade do processo de trabalho da Vigilância

Compreende-se como tarefas hercúleas, porém imprescidíveis, conciliar demandas e responsabilidades, muitas vezes com prazos para atendê-las, com momentos de estudos, reflexões, planejamento e avaliação.

A subdimensão Atenção Integral à Saúde obteve resultado "satisfatório", relacionado às questões sobre ações de proteção e promoção da saúde, prevenção e controle de riscos, agravos e doenças nos diversos níveis de atenção. Foram analisados aspectos sobre educação em saúde, ações de alerta e resposta a surtos e eventos de importância em saúde, inspeções, fiscalizações, emissão de licença sanitária, acolhimento e atendimento às notificações, denúncias e reclamações, busca ativa, controle de reservatórios, hospedeiros e vetores, vacinação e realização de inquéritos.

Há que se pensar na necessidade de harmonização de conhecimentos, entendimento do significado das atividades e um trabalho coordenado com os demais setores da Secretaria de Saúde e outros órgãos do governo, com apoio matricial da VS aos demais pontos de atenção da rede, no sentido de potencializar as ações, como mencionado nos resultados relativos aos gestores. Os profissionais valorizam ações educativas e a melhora dos canais de comunicação com o setor regulado, mesmo que seja para aumentar o espaço para denúncias. Sentem-se desconfortáveis com a lógica do trabalho das vigilâncias em "apagar 
incêndio". Acreditam que as ações de educação e prevenção competem à atenção básica, com apoio da VS.

A Participação, Controle Social e Satisfação do usuário foi avaliada como "satisfatória". Avaliouse particularidades a respeito de enfrentamento dos problemas sociais de maior expressão local, realização de ações conjuntas com a comunidade e debates sobre problemas locais de saúde e disponibilidade de canais de comunicação com os usuários.

Finalmente, serão destacados aspectos em relação ao instrumento utilizado na pesquisa autoavaliativa e aos resultados mais relevantes encontrados no estudo.

O instrumento trouxe um componente "formativo" ao incorporar a cada subdimensão um conjunto de explicações ou citações de alguns conceitos localizados nos recentes marcos legais do SUS, como, por exemplo, rede de atenção e redes temáticas, Relação Nacional de Ações e Serviços de Saúde (RENASES), Apoio Institucional, educação permanente, entre outros. Ou seja, mesmo que o respondente não estivesse familiarizado com o conceito, foi possível olhar para a prática e fazer uma reflexão se os temas apresentados já faziam ou não parte do seu repertório cotidiano. Há que se pensar em uma ferramenta mais sintética, sendo necessário rever a conveniência de algumas subdimensões serem avaliadas tanto por gestores como por trabalhadores.

A autoavaliação revelou pontos positivos e nós críticos sobre as práticas da VS como a incorporação do uso da informação no cotidiano de gestores e trabalhadores, a fragmentação das práticas interna à VS e externamente na rede de atenção à saúde, e problemas estruturais e organizativos a que estão submetidos os profissionais. Trabalhadores e gestores tiveram percepções diferentes sobre estes temas.

Percebe-se a falta de espaços para diálogos com outros pontos de atenção da rede de saúde, tanto na construção de projetos comuns de ações no território, quanto de apoio técnico ou matricial. Por um lado, os gestores cumprem os pactos interfederativos e pouco utilizam a informação no seu cotidiano e, por outro, os trabalhadores ficam imersos em seu universo de trabalho técnico, pouco reflexivo e com lacunas estruturais e organizativas. Ao mesmo tempo, produzem muitas informações que parecem ficar restritas ao domínio da alta direção ou aos técnicos de referência de determinados agravos.

Apropriar-se da informação produzida pelas equipes pode ser mobilizador para gestores $\mathrm{e}$ trabalhadores, no sentido de produzir uma prática avaliativa formativa. Acredita-se e espera-se, como Onoko-Campos e Furtado 7 , que "o envolvimento dos atores seja constante e ativo e o avaliador cumpra um papel de Facilitador. Espera-se que o processo avaliativo propicie assimilação, pelos participantes, de habilidades para compreender e utilizar melhor os resultados, bem como se envolver ou conduzir novas avaliações".

\section{Conclusões}

O questionário construído pelos pesquisadores para a autoavaliação de gestores e trabalhadores da VS mostrou-se suficiente e inovador ao abrir espaço às falas e reflexões dos mesmos. À semelhança do instrumento da AMAQ, incluiu elementos da avaliação formativa em seu conteúdo ao apresentar conceitos relacionados ao universo do SUS e da VS, mesmo àqueles não familiarizados com o tema.

A pesquisa apontou a autoavaliação como elemento relevante da avaliação sistemática das atividades desenvolvidas por gestores e trabalhadores. Seus resultados devem, necessariamente, ser postos em discussão na busca pela compreensão dos nós críticos e dos aspectos positivos da prática, com vistas a realizar mudanças e futuros contratos de gestão. Pode-se considerar a autoavaliação como mais um dispositivo de gestão, assim como os espaços coletivos, a educação permanente e o apoio institucional.

A autoavaliação mostrou-se potente para a análise das práticas e, diferentemente dos processos habituais de avaliação, pautados em indicadores quantitativos, incluiu os sujeitos e incorporou o olhar para si aos modos de fazer da Vigilância em Saúde. 


\section{Colaboradores}

MFG Vilela, DN Santos e B Kemp participaram de todas as etapas de elaboração do artigo.

\section{Referências}

1. Brasil. Ministério da Saúde (MS). Programa Nacional de Melhoria do Acesso e da Qualidade da Atenção Básica (PMAQ):manual instrutivo. Brasília: MS; 2012.

2. Brasil. Ministério da Saúde (MS). Autoavaliação para a Melhoria do Acesso e da Qualidade da Atenção Básica AMAQ. Brasília: MS; 2013.

3. Brasil. Ministério da Saúde (MS). Portaria $n^{\circ} 1.708 /$ GM/MS, de 16 de agosto de 2013. Regulamenta o Programa de Qualificação das Ações de Vigilância em Saúde - PQA-VS. Diário Oficial da União 2013; 19 ago.

4. Santos DN. Avaliação e contratualização na vigilância em saúde: caminhos possiveis [dissertação]. Campinas: Universidade Estadual de Campinas; 2016.

5. Brasil. Ministério da Saúde (MS). Portaria no 325/GM/ MS, de 21 de fevereiro de 2008. Estabelece prioridades, objetivos e metas do Pacto pela Vida para 2008, os indicadores de monitoramento e avaliação do Pacto pela Saúde e as orientações, prazos e diretrizes para a sua pactuação. Diário Oficial da União 2008; 22 fev.

6. Furtado JP. Um método construtivista para avaliação em saúde. Cien Saude Colet 2001; 6(1):165-182.

7. Onoko-Campos R, Furtado JP. Desafios da Avaliação de Programas e Serviços de Saúde. Campinas: Ed Unicamp; 2011.

8. Barbosa A. O PMAQ nas Equipes de Saúde da Família do Distrito Leste em Campinas SP [dissertação]. Campinas: Universidade Estadual de Campinas; 2016.

9. Moraes PN, Iguti AM. Avaliação do desempenho do trabalhador como forma peculiar de prescrição do trabalho. Saúde debate 2013; 37(98):416-426.

10. Vieira-da-Silva LM. Avaliação de Políticas e Programas de Saúde. Rio de Janeiro: Fiocruz; 2014.

11. Brasil. Ministério da Saúde (MS). Portaria no 1.378/ GM/MS, de 9 de Julho de 2013. Regulamenta as responsabilidades e define diretrizes para execução e financiamento das ações de Vigilância em Saúde pela União, Estados, Distrito Federal e Municípios, relativos ao Sistema Nacional de Vigilância em Saúde e Sistema Nacional de Vigilância Sanitária. Diário Oficial da União 2013; 9 jul.

12. Waldman EA. Vigilância como prática de Saúde Pública. In: Campos GWS, Minayo MC, organizadores. Tratado de Saúde Coletiva. Rio de Janeiro: Editora Hucitec; 2009. p. 487-528.

13. Thacker SB, Berkelman RL. Public health surveillance in the United States. Epidemiol Rev 1988; 10:64-90.

14. Brasil. Lei no 8.080, de 19 de setembro de 1990. Dispõe sobre as condições para a promoção, proteção e recuperação da saúde, a organização e o funcionamento dos serviços correspondentes e dá outras providências. Diário Oficial da União 1990; 20 set.

15. Vilela MFGV. Da 'moça da vigilância' ao núcleo de saúde coletiva na unidade básica de saúde: o que há de novo no modelo assistencial de Campinas? [tese]. Campinas: Universidade Estadual de Campinas; 2004.

16. Paim JS, Teixeira CLGM. Reorganização do sistema de vigilância epidemiológica na perspectiva do Sistema Único de Saúde (SUS). In: Anais do Seminário Nacional de Vigilância Epidemiológica; 1993; Brasília. p. 93-144. 
17. Mendes EV. Uma agenda para a saúde. $2^{a}$ ed. São Paulo: Hucitec; 1999.

18. Paim JS. Modelos de Atenção à Saúde no Brasil. In: Giovanella L. Políticas e Sistemas de Saúde no Brasil. 2a ed. Rio de Janeiro: Fiocruz; 2012. p. 459-491.

19. Campos GWS. Saúde pública e saúde coletiva: campo e núcleo de saberes e práticas. Cien Saude Colet 2000; 5(2):219-239.

20. Garcia RA, L'Abbate S. Institucionalização da Vigilância em Saúde de Campinas (SP) na perspectiva da Análise Institucional sócio-histórica. Saúde Debate 2015; 39(107):997-1007.

21. Silva GAP, Vieira-da-Silva LM. Vigilância em saúde: proposta de ferramenta para avaliar arranjos. Cad Saude Publica 2008; 24(11):2463-2475.

22. Costa E. O trabalhador de vigilância sanitária e a construção de uma nova vigilância: fiscal ou profissional de saúde? In: Costa E, organizadores. Vigilância Sanitária: desvendando o enigma. Salvador: EDUFBA; 2008. p. 7790.

23. Figueiredo A. Caminhos da Vigilância Sanitária Brasileira: proteger, vigiar e regular. São Paulo: Hucitec; 2016.

24. Silva Junior JB. Epidemiologia em serviço: uma avaliação de desempenho do Sistema Nacional de Vigilância em Saúde [tese]. Campinas: Universidade Estadual de Campinas; 2004.

25. Brasil. Ministério da Saúde (MS). Portaria n 14/GM/ MS, de 22 de agosto de 2013. Instituir Grupo de Trabalho para discussão e elaboração da Política Nacional de Vigilância em Saúde. Diário Oficial da União 2013; 23 ago.

26. Documento Base da Política Nacional de Vigilância em Saúde. Documento elaborado pelo GT-PNVS, instituído por meio da Portaria SVS/MS no 14/2013. Brasília: Ministério da Saúde, Conass, Conasems; 2014).

27. XXIX Congresso de Secretários Municipais de Saúde do Estado de São Paulo. 2015 mar 18-20; Campos do Jordão; São Paulo; 2015. [acessado 2017 jun 12]. Diponível em: http://www.cosemssp.org.br/congresso2015/ texto.php?id_texto=1

28. Oliveira TGHD. A política e as diretrizes da Vigilância em saúde no SUS. Palestra em Curso In: XXIX Congresso de Secretários Municipais de Saúde do estado de São Paulo; 201520 de Março; Campos de Jordão, São Paulo.

29. Brasil. Ministério da Saúde (MS). Portaria $n^{\circ} 1.017 /$ GM/MS, de 11 de maio de 2016. Convoca a $1^{\text {a }}$ Conferência Nacional de Vigilância em Saúde. Diário Oficial da União 2016; 13 maio.

30. Brasil. Conselho Nacional de Saúde. Resolução no 535 , de 19 de agosto de 2016. Aprovar o Regimento da $1^{\text {a }}$ Conferência Nacional de Vigilância em Saúde. Diário Oficial da União 2016; 11 nov.

31. Minayo MCS. O desafio do conhecimento: pesquisa qualitativa em saúde. 12a ed. São Paulo: Hucitec; 2010.

32. Bardin L. Análise de conteúdo. Lisboa: Edições 70; 1977.

33. Brasil. Ministério da Saúde (MS). Decreto ${ }^{\circ} 7.508$, de 28 de junho de 2011. Regulamenta a Lei no 8.080 , de 19 de setembro de 1990, para dispor sobre a organização do Sistema Único de Saúde - SUS, o planejamento da saúde, a assistência à saúde e a articulação interfederativa, e dá outras providências. Diário Oficial da União 2011; 29 jun.
34. Bobbio N. Dicionário de política. Brasília: Universidade de Brasília; 1998. Vol. 1.

35. Brasil. Ministério da Saúde (MS). Portaria n 2.488/ GM/MS, de 21 de outubro de 2011. Aprova a Política Nacional de Atenção Básica, estabelecendo a revisão de diretrizes e normas para a organização da Atenção Básica, para a Estratégia Saúde da Família (ESF) e o Programa de Agentes Comunitários de Saúde (PACS). Diário Oficial da União 2011; 21 out.

36. Brasil. Conselho Nacional de Secretários Municipais de Saúde (CONASS). A Atenção Primária e as Redes de Atenção à Saúde. Brasília: CONASS; 2015.

37. Fernandes RZS, Vilela MFG. Estratégias de integração das práticas assistenciais de saúde e vigilância sanitária no contexto de implementação da Rede Cegonha. Cien Saude Colet 2014; 19(11):4457-4466.

38. Campos GWS. Um método para análise e cogestão de coletivos. São Paulo: Hucitec; 2000.

39. Oliveira MM, Campos GWS. Apoios matricial e institucional: analisando suas construções. Cien Saude Colet 2015; 20(1):229-238.

40. Brasil. Emenda Constitucional no 95, de 15 de dezembro de 2016. Altera o ato das disposições constitucionais transitórias, para instituir o novo regime fiscal, e dá outras providências. Diário Oficial da União 2016; $15 \mathrm{dez}$.

41. Von Zuben A, Cordeiro MRD. Dificuldades na execução das diretrizes do Programa de Vigilância e Controle da Leishmaniose Visceral em grandes municípios brasileiros. Cad Saude Publica 2016; 32(6):1-11.

42. Drumond JR M. Epidemiologia nos municípios, muito além das normas. São Paulo: Hucitec; 2003.

43. Brasil. Ministério da Saúde (MS). Diretrizes nacionais da vigilância em saúde. Brasília: MS; 2010.

44. Brasil. Ministério da Saúde (MS). Fundação Nacional da Saúde. Projeto VIGISUS: Estruturação do Sistema Nacional de Vigilância em saúde. Brasília: MS; 1998.

45. Brasil. Ministério da Saúde (MS). Projeto VIGISUS II: Modernização do Sistema Nacional de Vigilância em saúde. Brasília: MS; 2003.

46. Brito SMF. Avaliação de estados e municípios na execução do Projeto Vigisus II: limites e possibilidades [dissertação]. Salvador: Universidade Federal da Bahia; 2008.

47. Brasil. Ministério da Saúde (MS). Datasus. [acessado 2017 jun 12]. Diponível em: http://datasus.saude.gov. $\mathrm{br} /$ sistemas-e-aplicativos

48. Brasil. Ministério da Saúde (MS). Política Nacional de Informação e Informática em Saúde. Brasília: MS; 2016.

49. White KL. Information for healthcare: in epidemiological perspective. Inquiry 1980; 17:296-312.

Artigo apresentado em 30/05/2017

Aprovado em 26/06/2017

Versão final apresentado em 17/07/2017 